\title{
Signal processing method for data with simultaneous recording of auditory brainstem response, middle latency response, and slow vertex response
}

\author{
Katuhiro Maki ${ }^{1, *}$, Tomoharu Ishikawa ${ }^{2}$ and Kazuki Kawakami ${ }^{2}$ \\ ${ }^{1}$ Faculty of Human Informatics, Aichi Shukutoku University, 2-9 Katahira, Nagakute, 480-1197 Japan \\ ${ }^{2}$ Graduate School of Regional Development and Creativity, Utsunomiya University, \\ 7-1-2 Yoto, Utsunomiya, 321-8585 Japan
}

(Received 16 August 2019, Accepted for publication 13 September 2019)

Keywords: Evoked potential, Cortical response, Auditory pathway, Bandpass filter, Bartlett window PACS number: 43.64.Qh, 43.64.Ri, 43.58.Kr [doi:10.1250/ast.41.517]

\section{Introduction}

Various types of auditory evoked response can be recorded from the human scalp, such as cochlear microphonics (CM), auditory-nerve compound action potential (CAP), auditory brainstem response (ABR), auditory middle latency response (MLR), auditory slow vertex response (SVR), and auditory contingent negative variation (CNV). Among these, ABR, MLR, and SVR can be simultaneously recorded with the same electrode arrangement on the scalp [1-9]. Additionally, the latencies of these responses increase, in the order of ABR, MLR, and SVR [1-10], and that they mostly reflect the neural activity in the brainstem, in both the brainstem and the auditory cortex, and in the auditory cortex, respectively. Thus, simultaneous recording of ABR, MLR, and SVR has the potential to be used for functional elucidation and clinical inspection of the auditory pathway from the brainstem to the auditory cortex [2-9].

Since the frequency bands in which ABR, MLR, and SVR appear are different, a bandpass filter with different passbands is applied to measurement data of these responses to observe them clearly [1-10]. However, when this filtering method is applied to data obtained by simultaneous recording of ABR, MLR, and SVR, these responses cannot be observed on one continuous waveform in the same coordinate axes with respect to time and amplitude, because the passbands are different from each other. Therefore, the components of ABR, MLR, and SVR cannot be compared directly. For example, the peak amplitudes of the components of ABR, MLR, and SVR are smaller after filtering than before filtering. The reduction in amplitude caused by this filtering depends on the bandwidth and cutoff frequency of the filter applied to ABR, MLR, and SVR. Thus, it is not preferable to compare amplitudes between them because they include the influence of filter parameters. In this study, we propose a method to observe ABR, MLR, and SVR on one continuous waveform in the same coordinate axes by smoothing the measurement data using a window function with a time-variant window size.

\section{Experimental methods}

2.1. Recording auditory evoked responses

The subjects were four university students (male) with

\footnotetext{
*e-mail: maki-ns@umin.ac.jp
}

normal hearing from 21 to 23 years of age. A rectangular click with a duration of $0.1 \mathrm{~ms}$ was used as a stimulus. The click sound was presented to the subjects binaurally at a sound pressure level of $60 \mathrm{~dB}$ above the hearing threshold. The stimulus was presented 1,000 times at an interval of $500 \mathrm{~ms}$ via an in-ear-type earphone (ER4-SR, Etymotic Research).

Each subject sat in a quiet, dark room and closed his eyes during the observation. The A-weighted sound pressure level of background noise in the room was $30 \mathrm{~dB}$. The active electrode was placed on the vertex of the head $(\mathrm{Cz})$. Two reference electrodes were placed, one on the left earlobe and the other on the right earlobe. A ground electrode was placed on the lower forehead. The induced potential was recorded by a computer at a sampling frequency $\left(F_{\mathrm{s}}\right)$ of $48 \mathrm{kHz}$ with 24-bit quantization via an audio interface (Digiface USB, RME) after using an amplifier (Biotop mini, East Medic Corp) to boost the 0.53 to $3,000 \mathrm{~Hz}$ frequency range. The response to the repeatedly presented stimulus for each subject was averaged into a time window of $500 \mathrm{~ms}$. This averaged response was used as preprocessing measurement data in Sect. 2.2.

2.2. Proposed signal processing method

The preprocessing measurement data including ABR, MLR, and SVR were smoothed by applying a window function with a time-varying window length, instead of using a bandpass filter. Bartlett (triangular) and Hamming windows were used as the window functions.

The Bartlett window is given by Eq. (1).

$$
B(t)= \begin{cases}1-\frac{|t|}{T} & (|t|<T) \\ 0 & (|t|>T)\end{cases}
$$

Here, $t$ represents time. $T$ represents the window length, which is usually set independent of time. In this study, smoothing using function with a time-varying window length was realized by making $T$ as a function of time $t_{\mathrm{c}}$ (in unit of seconds) from the stimulus onset. Although the window length $T\left(t_{\mathrm{c}}\right)$ can be set in various ways, we chose a simple function, $T\left(t_{\mathrm{c}}\right)=a t_{\mathrm{c}}+b$, in our first attempt. In the function of $T\left(t_{\mathrm{c}}\right), a$ and $b$ are positive coefficients. In this study, 0.05 and 0.00003 were used as the values of $a$ and $b$, respectively. The parameter value of $a(0.05)$ was chosen so that the result of applying the proposed method is close to the results of 


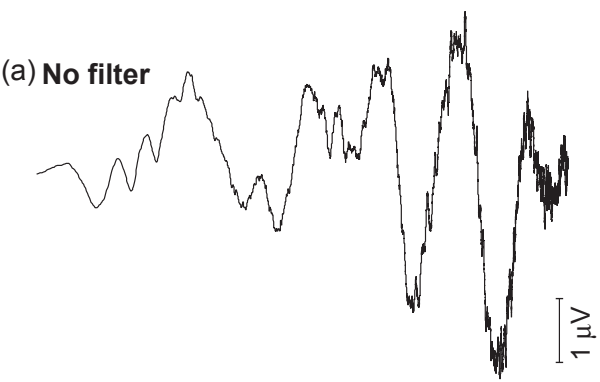

(b) $50 \mathrm{~Hz}-3000 \mathrm{~Hz}$

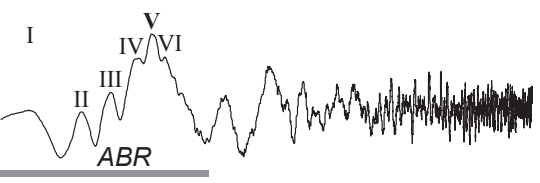

(c) $10 \mathrm{~Hz}-300 \mathrm{~Hz}$
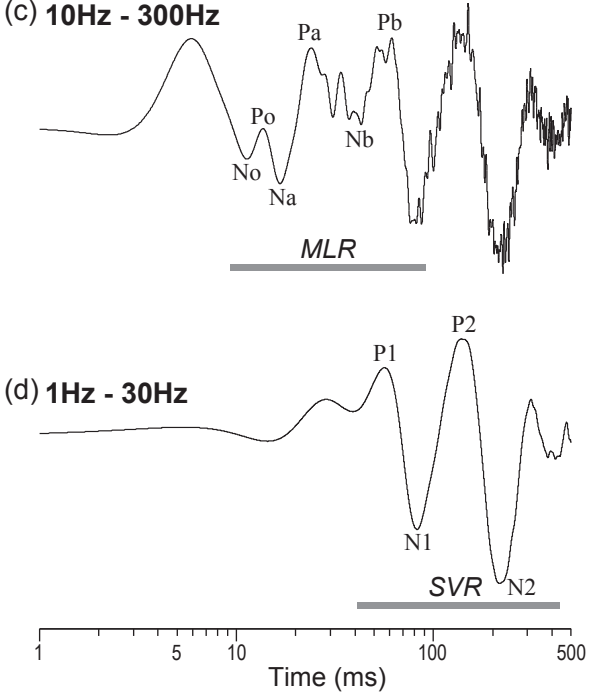

Fig. 1 Results of applying a bandpass filter with different passbands to measurement data including ABR, MLR, and SVR. Panel (a) shows the preprocessing measurement data, which is the original waveform without applying a bandpass filter. Panels (b), (c), and (d) show filtering results with passbands of 50$3,000 \mathrm{~Hz}, 10-300 \mathrm{~Hz}$, and $1-30 \mathrm{~Hz}$, respectively. The time axis in each panel is represented in a logarithmic scale because the latencies of ABR, MLR, and SVR are largely different. The scales of the vertical axes in (a) to (d) are the same. ABR, MLR, and SVR observed in different time ranges are indicated by horizontal bars.

applying bandpass filters with passbands of $50-3,000 \mathrm{~Hz}, 10$ $300 \mathrm{~Hz}$, and $1-30 \mathrm{~Hz}$ to ABR, MLR, and SVR, respectively (see Figs. 1 and 3$)$. The value of $b(0.00003)$ was determined so that the window length had at least one sample at a sampling frequency of $48 \mathrm{kHz}$.

\section{Results}

Figure 1(a) shows an example of preprocessing measurement data including ABR, MLR, and SVR. Figures 1(b)-1(d) show the results of applying the bandpass filter with different passbands to the data from Fig. 1(a) to observe ABR, MLR, and SVR more clearly without fine amplitude fluctuation. This filtering method has commonly been used in previous studies, although there are not many examples of simultaneous recording of ABR, MLR, and SVR (see Introduction).

When the bandpass filter of 50 to $3,000 \mathrm{~Hz}$ was applied (Fig. 1(b)), typical wave-I to wave-IV of ABR were observed, which were similar to the original data shown in Fig. 1(a), except that the amplitude was reduced considerably after $10 \mathrm{~ms}$. When the passband was 10 to $300 \mathrm{~Hz}$ (Fig. 1(c)), the response components of MLR ( $\mathrm{No}, \mathrm{Po}, \mathrm{Na}, \mathrm{Pa}, \mathrm{Nb}$, and $\mathrm{Pb}$ ) were observed from 10 to $100 \mathrm{~ms}$. However, wave-I to waveIV of ABR (Fig. 1(b)) integrated into a single peak. Conversely, fine amplitude fluctuation remained after $100 \mathrm{~ms}$. When the passband was 1 to $30 \mathrm{~Hz}$, the response components of SVR (P1, N1, P2 and N2) were observed without fine amplitude fluctuations, but the response components of MLR were attenuated and those of ABR almost disappeared.

When the bandpass filter was used to observe ABR, MLR, and SVR, these responses could not be observed on one continuous waveform in the same coordinate axes of time and amplitude. This made it impossible to directly compare the amplitudes of response components observed in ABR, MLR, and SVR. Actually, the peak value of ABR wave-V was hardly affected by filtering (Fig. 1(b)), but the peak value of the P2 component of SVR (Fig. 1(d)) was attenuated at a level that can be visually recognized than that before the filtering (Fig. 1(a)). That is, since the peak value of the P2 component includes the influence of filter parameters such as the cutoff frequency, it is not preferable to directly compare it with the amplitude of ABR. Moreover, although MLR Pb (Fig. 1(c)) and SVR P1 (Fig. 1(d)) originate from the same peak component of the original waveform (Fig. 1(a)), it has been pointed out that analysis using a bandpass filter may treat each as a different component [1].

The result of applying the proposed method to the preprocessing measurement data (Fig. 1(a)) is shown in Fig. 2(a). In this case, the Bartlett window function was used (see Sect. 2.2). The waveforms of ABR, MLR, and SVR obtained from the proposed method shown by the black line in Fig. 2(a) were similar to the those of their respective parts shown in Figs. 1(b)-1(d), which were the results of applying the bandpass filter with different passbands. Also, when applying the proposed method, ABR, MLR, and SVR were observed on one continuous waveform in the same time and amplitude axes. Furthermore, the waveform obtained by the proposed method (black line) followed the temporal change in the original waveform (red line) with the appropriate time resolution. This can be roughly confirmed the observation that the difference between the processed data (black line) and the original data (red line) is distributed symmetrically with 0 as a boundary (Figs. 2(b) and 2(e)). This was also true for the data from the other subject shown in Figs. 2(d) and 2(f). The coefficients of skewness for the data in Figs. 2(e) and 2(f) were 0.084 and -0.06 , respectively, and those of the other two subjects were -0.07 and 0.048 . This indicates the distributions were nearly symmetrical.

The appearance of the response components of ABR, MLR, and SVR were partially different and depended on the subject (cf. Figs. 2(a) and 2(c)). For example, for subject KK 

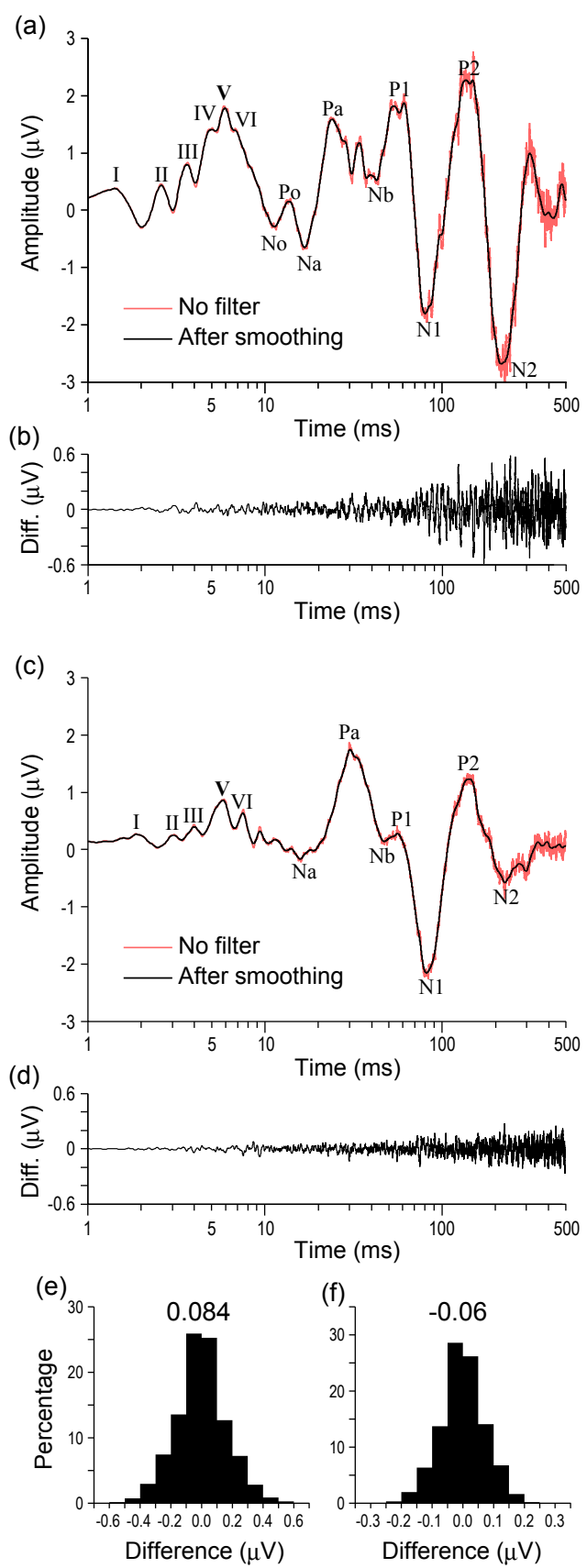

Fig. 2 Results of applying the proposed method to the preprocessing measurement data. Red lines in (a) and (c) indicate preprocessing measurement data of two subjects (KK and TI, respectively). The red line in (a) is the same as the data in Fig. 1(a). Black lines in (a) and (c) represent the results from the proposed method. Panels (b) and (d) show the differences between before and after applying the proposed method. Panels (e) and (f) show histograms of the data in (b) and (d), respectively. The numbers shown at the top of panels (e) and (f) are coefficients of skewness and indicate the degree of symmetry.

(Fig. 2(a)), the ABR wave-IV and MLR Po component were observed, but not for subject TI (Fig. 2(c)). However, regardless of the subject, the proposed method succeeded in

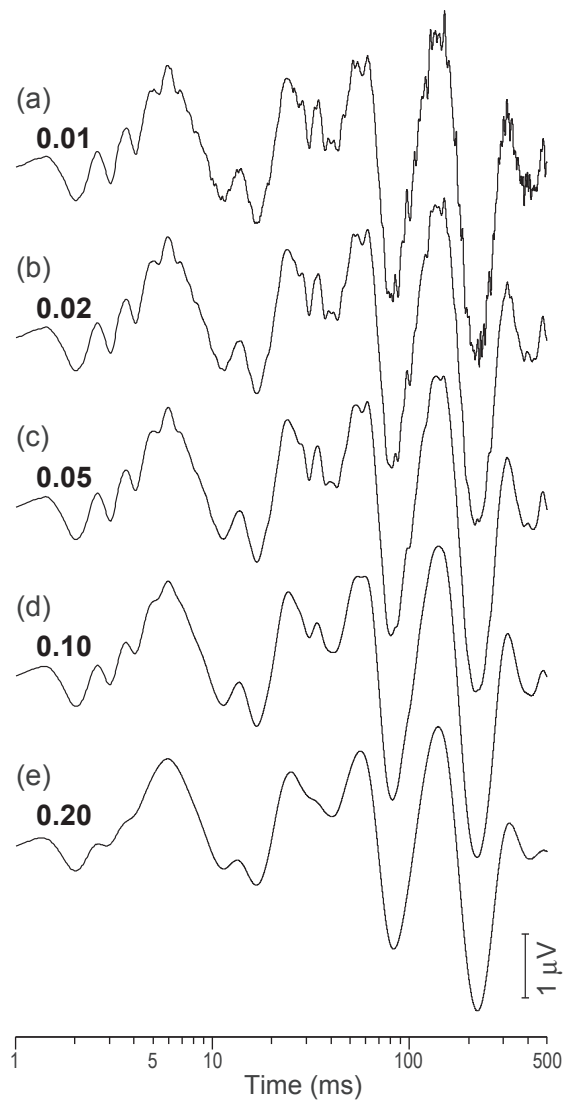

Fig. 3 Changes in waveform when the value of parameter $a$ is changed in the proposed method. Panels (a) to (d) represent the smoothing results when $a$ (see Sect. 2.2) is changed from 0.01 to 0.20 , which defines the length of the Bartlett window. Each number represents the value of $a$. The waveform shown in (c) is the same as the waveform shown by the black line in Fig. 2(a).

smoothing the data to remove the fine amplitude fluctuations that disturb observation of the response components.

Figure 3 shows changes in the waveform after smoothing by the proposed method when the parameter $a$ (see Sect. 2.2), which defines the time-varying window length, is changed. From Fig. 3, it can be seen that the visibility of each ABR waveform deteriorates when $a$ is greater than 0.10 . On the other hand, when $a$ is less than 0.02 , fine amplitude fluctuation remains in the SVR, particularly after $100 \mathrm{~ms}$. Thus, it can be concluded that the value of $a$ is preferably set to around 0.05 .

Even when using the Hamming window for the function, the result was almost the same as that when using the Bartlett window when the length of the Hamming window $N\left(t_{\mathrm{c}}\right)$ (unit is the number of samples) was the same as that of the Bartlett window, which was given by $N\left(t_{\mathrm{c}}\right)=2 T\left(t_{\mathrm{c}}\right) \cdot F_{\mathrm{s}}$.

\section{Discussion}

We showed that ABR, MLR, and SVR can be analyzed by smoothing the measurement data using a time-variant window function, instead of using a bandpass filter with different passbands. Moreover, we clarified the parameter values of the window functions for which the smoothing process was 
successfully applied regardless of the subject. Thus, the proposed method can be applied to measurement data without the need to adjust the parameter values. Since the type of window function had almost no influence on the results, when fine adjustment is necessary, it is best to change the function that determines a time-varying window length and its parameter values.

In the analysis of ABR, some studies have adopted analytical methods that divide ABR into fast and slow waves $[11,12]$. The data processing method proposed in this study can extract slow waves by adjusting the window length of the function (see Fig. 3(e)) but cannot extract fast waves. A limitation of the proposed method is that it cannot analyze the fast waves of $\mathrm{ABR}$, but it can be used to directly compare ABR, MLR, and SVR, which are composed of different frequency components and exhibit different latencies, which is an advantage of the proposed method.

From the viewpoint of signal processing, a moving average on the time axis using a time-varying window length has an effect similar to that of a low-pass filter applied while changing the cutoff frequency of the filter over time [13]. However, the design of the time-varying window length in the moving average (see Sect. 2.2) is considered to be intuitive and simpler than designing the filter coefficient at each time in the low-pass filter. Thus, it is better to use the proposed method for data of auditory evoked potential.

\section{Conclusion}

We propose a method to observe ABR, MLR, and SVR on one continuous waveform in the same coordinate axes by performing data smoothing and changing the smoothing width with time. This enables the direct comparison of ABR, MLR, and SVR with respect to time and amplitude, which may be useful for functional elucidation of the auditory pathway from the auditory brainstem to the auditory cortex.

\section{Acknowledgments}

This work was supported by JSPS KAKENHI Grant Number JP17K19995.

\section{References}

[1] G. Ichikawa, N. Uehara, S. Ishikawa, H. Saito, T. Yamakawa and Y. Ehara, "A consideration on continuous recording of auditory evoked responses," Audiology, 32, 103-111 (1989) (in Japanese).

[2] S. M. Mason and D. H. Mellor, "Brain-stem, middle latency and late cortical evoked potentials in children with speech and language disorders," Electroencephalogr. Clin. Neurophysiol., 59, 297-309 (1984).

[3] J. J. Rotteveel, E. J. Colon, S. L. Notermans, G. B. Stoelinga and Y. M. Visco, "The central auditory conduction at term date and three months after birth. I. Composite group averages of brainstem (ABR), middle latency (MLR) and auditory cortical responses (ACR)," Scand. Audiol., 14, 179-186 (1985).

[4] B. Cone-Wesson, D. Kurtzberg and H. G. Vaughan Jr., "Electrophysiologic assessment of auditory pathways in high risk infants," Int. J. Pediatr. Otorhinolaryngol., 14, 203-214 (1987).

[5] G. Japaridze, D. Kvernadze, T. Geladze and Z. Kevanishvili, "Effects of carbamazepine on auditory brainstem response, middle-latency response, and slow cortical potential in epileptic patients," Epilepsia, 34, 1105-1109 (1993).

[6] G. Japaridze, R. Shakarishvili and Z. Kevanishvili, "Auditory brainstem, middle-latency, and slow cortical responses in multiple sclerosis," Acta Neurol. Scand., 106, 47-53 (2002).

[7] J. B. Firszt, R. D. Chambers, N. Kraus and R. M. Reeder, "Neurophysiology of cochlear implant users I: Effects of stimulus current level and electrode site on the electrical ABR, MLR, and N1-P2 response," Ear Hear., 23, 502-515 (2002).

[8] S. C. Purdy, A. S. Kelly and M. G. Davies, "Auditory brainstem response, middle latency response, and late cortical evoked potentials in children with learning disabilities," J. Am. Acad. Audiol., 13, 367-382 (2002).

[9] A. Dixit, N. Vaney and O. P. Tandon, "Effect of caffeine on central auditory pathways: An evoked potential study," Hear Res., 220, 61-66 (2006).

[10] T. W. Picton, Human Auditory Evoked Potentials (Plural Publishing, San Diego, 2011), pp. 2-122.

[11] N. Takagi, T. Suzuki and K. Kobayashi, "Effect of tone-burst frequency on fast and slow components of auditory brain-stem response," Scand. Audiol., 14, 75-79 (1985).

[12] T. Suzuki, K. Kobayashi and N. Takagi, "Effects of stimulus repetition rate on slow and fast components of auditory brainstem responses," Electroencephalogr. Clin. Neurophysiol., 65, 150-156 (1986).

[13] S. W. Smith, Digital Signal Processing: A Practical Guide for Engineers and Scientists (Newnes, Burlington, 2002), pp. 277-284. 